\begin{tabular}{ll} 
Complementing your partner & 226 \\
\hline The big picture & 226 \\
\hline Guilt by association & 228 \\
\hline Don't fence me in & 229 \\
\hline Box 1: Information overload & 229 \\
\hline
\end{tabular}

\title{
Cell signaling branches out
}

\author{
Directly or indirectly, most cell biologists study cell signaling, a field whose complexity has grown \\ geometrically with recent advances in molecular biology. Combining refinements of older techniques with \\ innovative new methods can help researchers traverse this rapidly expanding field. Alan Dove reports.
}

Ask a cell biologist to explain signal transduction, and you are in for a long story. The science of understanding how individual cells sense their environments and respond to stimuli fills library shelves, occupies whole departments of colleges and inspires the careers of thousands of researchers around the world. Even so, the field sometimes seems woefully understaffed. The advent of whole-genome sequencing and gene-expression profiling revealed what most biologists already suspected: we are just beginning to understand cell signaling.

Although there will undoubtedly be more surprises in the coming years, the past few decades have revealed some common themes in signaling. For example, cells rely heavily on surface receptor proteins to communicate with the outside world, and a large proportion of medically important signals flows through receptors that are coupled to effector molecules called G proteins. Inside the cell, post-translational protein modification is standard currency. Information flow often entails an enzyme finding a specific target protein and attaching or removing phosphates, lipid groups, or other chemical structures. The modified target commonly goes on to modify other targets and so on through baroque cascades of interactions.

Besides serving as a powerful exhibit in arguments against the idea of a rational creator, the clunky complexity of cell signaling guarantees years of future work for cell biologists. Fortunately, the tools for probing cell signaling have evolved rapidly in recent years, and numerous companies now offer reagents, kits and services to make these studies easier. Meanwhile, researchers continue to develop innovative new techniques for probing signaling pathways, sometimes simply by com-
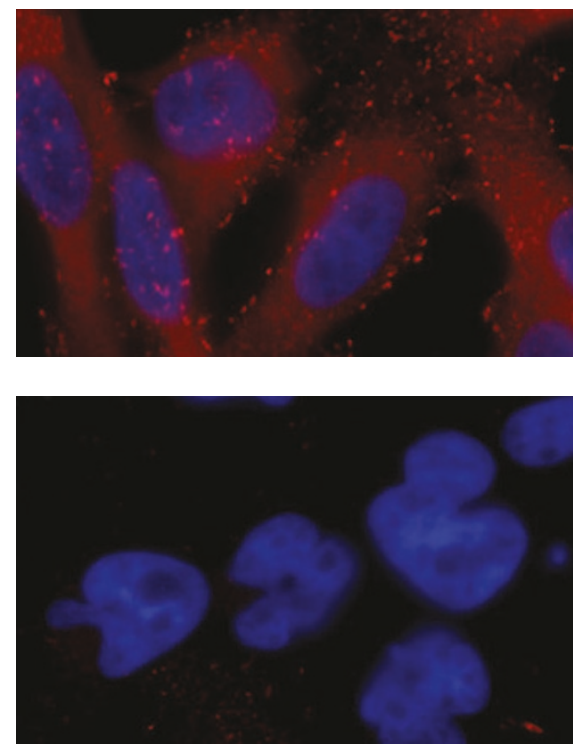

Antibody providers offer kits for easier experimental controls. Here, a monoclonal antibody stains the focal adhesion kinase (FAK) signaling molecule (top), and an siRNA provided with the antibody shuts off FAK expression to confirm that the staining is specific (bottom). (Courtesy of Serologicals.)

bining existing technologies in new and sophisticated ways.

\section{Finding your non-self}

Many cellular signals ultimately lead to changes in gene expression, but the signals themselves almost always travel through proteins, forcing signal transduction researchers into the challenging terrain of protein chemistry. Fortunately, dozens of reagent suppliers cater to the protein analysis needs of signal transduction workers, especially when it comes to providing essential tools like antibodies.

Traditionally, investigators use antibodies for western blots, protein purification and microscopic techniques like immuno- histochemistry and immunocytochemistry. Because antibodies are produced by living organisms, they are nearly impossible to standardize, but most manufacturers will provide detailed descriptions of their products' binding properties and references to related peer-reviewed papers.

"What really drives sales in this business are publications and applications, so if someone has used a particular antibody in a paper, people will want to use that same antibody ... because it has been validated already by a researcher that this one works in this particular system," says Greg Moore, director of scientific sourcing and new technologies at Upstate.

Using the same antibodies as one's competitors and colleagues also makes comparisons between studies easier, so for most projects the first stop on the shopping trip should be the peer-reviewed literature. When the literature on a particular protein includes multiple antibodies, researchers should choose based on features and intended uses; some antibodies are best for identifying denatured signaling proteins on a western blot, whereas others are better for tagging native proteins in immunocytochemistry.

Most antibody makers also provide related products and kits, and many, including Upstate, even offer to do the work for some projects. A laboratory that does not normally analyze cytokine expression profiles, for example, might be better off sending a serum sample to a professional lab and receiving the results electronically than buying the reagents and learning how to do the analysis themselves.

Western blots can reveal changes in the expression level of a signaling protein, immunocytochemistry can show whether the protein moves within the cell and phosphospecific antibodies can indi- 

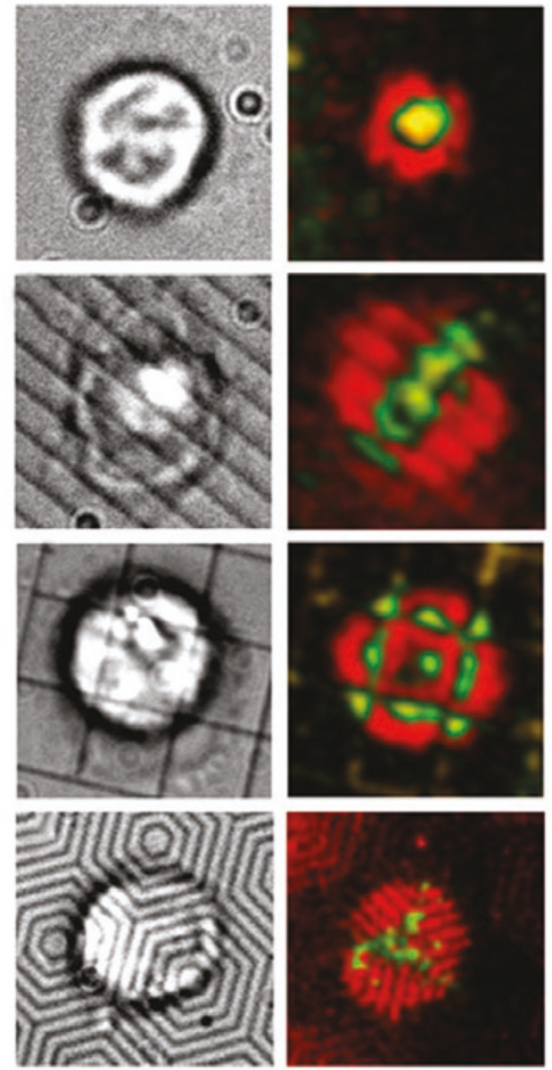

Nanoscale fences restrict the movements of immunological synapse complexes as shown in brightfield (left) and fluorescence (right) microscopy. Fluorescent labels stain ICAM red, T-cell receptors green and MHC molecules yellow in the right panels. Progressively more complex barriers produce a range of 'spatial mutations' affecting signaling efficiency. (Courtesy of J. Groves.)

cate whether it is phosphorylated, one of the most common mechanisms of signal transduction. Researchers have traditionally done these experiments separately, but at least one company is now integrating several popular signal transduction assays and also tying the information to previously published work.

Instead of probing a gel with a single antibody, "we can analyze multiple targets on a single SDS-PAGE ... the size of a minigel," explains Steven Pelech, president and founder of Kinexus. Choosing from a pool of validated antibodies, the company can identify the phosphorylation patterns and expression levels of as many as 40 known proteins in a cell extract simultaneously. With a new microarray-based product, Kinexus can simultaneously analyze all of the proteins for which they have validated antibodies, currently about 600 .

Indeed, the universe of available anti- bodies has become the main limiting factor in expanding the system even further. "Because we've actually tested a lot of these antibodies from companies, and we're running out of antibodies, we're actually thinking of forming a consortium where we're going to codevelop antibodies with a lot of our suppliers," says Pelech.

To use this approach, researchers need only send their samples to Kinexus. For about $\$ 25$ per protein, the company will report the expression and phosphorylation levels of up to 40 proteins in each sample with the mini-gel technique. The new microarray service will drive the price down to about $\$ 1$ per protein, for data on 600 targets. In addition, customers gain access to a database that includes data from other customers' experiments, allowing easy comparisons across systems. Researchers working on proprietary projects can keep their data out of the company's database, but that doubles the price of the assay.

\section{Complementing your partner}

Antibodies are an important way to probe cell signaling, but of course they are not the only way, and some of the elegant strategies originally developed for other types of studies are now finding new applications in signal transduction labs. For example, DiscoveRx initially marketed its enzyme fragment complementation system to pharmaceutical companies, but academic researchers are increasingly applying it in more basic research.

The technique uses a $\beta$-galactosidase enzyme that has been cut into two pieces. Separately, each piece is inactive, but when they come together, they can complement each other and break down their substrates. Feeding an artificial substrate to the reconstituted enzyme yields a chemiluminescent signal. "It's very highly sensitive, and it's also very amenable to highthroughput detection," says Richard Eglen, the chief scientific officer at DiscoveRx.

Theoretically, one could use the system to look at interactions between proteins, but Eglen warns that the $\beta$-galactosidase fragments have a high affinity for each other, raising the risk of artificial results. Instead, many researchers are using the technique to measure protein levels in a cell, or movement to a specific compartment within the cell. For example, expressing one fragment of the enzyme with a nuclear localization signal, and fusing the other fragment to a protein of interest, will make the nucleus glow whenever the test protein moves there. Besides nuclear translocation, "we have data showing it can move from cytoplasm to the membrane, we can measure also secretion of proteins from the cytoplasm to the cell exterior and also presentation of proteins on the cell surface," says Eglen.

Cell signaling researchers have already used the DiscoveRx system to track the movements and levels of several signaling proteins in response to stimuli, particularly in the measurement of changes in cAMP in response to $G$ protein-coupled receptor activation. The technique also provides a good alternative to traditional antibody-based assays, at least for some applications. "We've used this to be able to measure expression of proteins using a gel system. This allows people to rapidly undertake gel electrophoresis and visualize the protein, particularly at low expression levels. In principle, it is very much like a western blot but without the use of antibodies, because one is detecting it by complementation," says Eglen.

\section{The big picture}

Even with simpler, more sensitive assays, most cell signaling studies still require a lot of painstaking protein chemistry and slow, serial tracing of each step in a signaling pathway. Proteomics promises a different approach, tracing protein interactions in parallel to construct maps of all of the signals in a cell at a given time. Although much of that promise remains untapped, at least one off-the-shelf proteomics system is now rapidly gaining a following among signal transduction researchers.

In theory, one could learn a lot about cell signaling by purifying every protein expressed in a particular type of cell, spotting the purified proteins onto an array representing that cell's entire proteome, and then washing purified test proteins over the array to see where they stick. The assay itself would be quick and easy, but as any biochemist can attest, purifying all of those proteins would be a Herculean task.

"The hard part was getting the arrays," says Michael Snyder, professor of molecular, cellular and developmental biology at Yale University. A few years ago, Snyder and his colleagues developed techniques for high-throughput protein purification, then purified thousands of proteins from Saccharomyces cerevisiae and spotted 
them onto arrays. Invitrogen now sells the yeast arrays as well as a human version, and even though neither array contains a complete proteome yet, the company is actively developing both products. The human chip currently holds 5,000 different proteins, but larger versions are in the works. With an estimated human proteome of 25,000 proteins, Snyder says क "you could probably get the whole thing on two chips ... the whole human proteome spotted in duplicate."

Besides simple binding studies, the chips can also be used to analyze signaling reactions directly. For example, Snyder and his colleagues recently incubated yeast arrays with purified signaling proteins and found the likely substrates for 87 of the 122 known yeast protein kinases. "It's a very high-throughput, large-scale meth- od for finding lots of targets and lots of substrates, and you find lots of wonderful surprises," says Snyder. The scan revealed thousands of new leads, many of which have now been validated. The researchers are now developing methods to do similar proteome-wide scans for the targets of other enzymatic activities, such as methylation, acetylation and ubiquitination.

With the arrays now commercially available, any biochemistry laboratory seeking to generate a flood of new signaling data (see Box 1) can now use the technique. The system accomodates both radioactive and fluorescent assays, so the equipment for reading the arrays could be as simple as some X-ray film and a magnifier.

Though Invitrogen is the only company offering Snyder's technology, BD Biosciences, Pierce and other major suppliers offer complementary proteome-scale arrays for probing cell signaling, usually with an emphasis on medically important signaling pathways.

\section{Guilt by association}

Many cell signaling researchers focus on phosphorylation and other protein modifications, partly because these processes are important, but also because there are sophisticated methods to study them. Other cell signaling mechanisms have been harder to analyze. Over the past several years, cell biologists have discovered that the formation, movement and dissolution of protein complexes on the cell surface is extremely important in transducing many cellular signals, but tracking these rapidlychanging processes can be difficult.

Anja Nohe, assistant professor in the Institute of Molecular Biophysics at the University of Maine is pioneering a new technique that could help change that. Using tools most cell biology laboratories probably already own, Nohe and her colleagues can quantify the changes in protein aggregations on cell surfaces under different experimental conditions. The method, called family of images correlation spectroscopy (FICS) is "a fairly new technique, which is related to fluorescence correlation spectroscopy [FCS]. The difference is that we do the correlation of images, while FCS uses a certain volume to do the correlation analysis," says Nohe.

To do FICS, a researcher needs only a confocal fluorescence microscope and the ability to stain the proteins of interest, usually with fluorescently labeled anti- 
bodies. A healthy tolerance for tedium is also useful, as the method requires finding and imaging flat areas on the membranes of at least 40 cells in each sample, and each experiment and control must be done in triplicate. That translates to a lot of time spent staring through a microscope. The data are then fed into standard statistical analysis software to calculate a correlation

function, and changes in the amplitude of that function indicate protein movement, clustering and diffusion.

"You use this if you want to, for example, ... measure changes of proteins on the plasma membranes, if [a receptor has] to aggregate in caveolae or clathrincoated pits," says Nohe. So far, only three labs are using FICS on a regular basis, but they have already made some fundamental discoveries. Nohe and her colleagues have shown that the receptors for bone morphogenetic protein cluster in special membrane domains that reshuffle as the signal is regulated.

The main limitation of FICS seems to be a lack of patience with the painstaking imaging process. "I have gotten a lot of inquiries. The problem for me is most of them just want me to do the experiment. It's nice, but ... there's only so much I can do," says Nohe, who already has a full plate of collaborations with other laboratories. She points out, however, that suitable fluorescence microscopes and imaging equipment are available from manufacturers like Bio-Rad and Olympus, and a standard statistical application like Systat Software's SigmaPlot can be used to calculate correlation functions.

\section{Don't fence me in}

If a particular receptor clustering pattern seems to be important for signaling, the next logical experiment is to perturb the interaction and see whether the signal is affected. Traditionally, researchers have done this by engineering mutations in the receptor gene, by chemically modifying the receptor protein or by using competitive binding inhibitors like receptor-specific antibodies. Each approach has pitfalls, though, and any type of chemical alteration raises the possibility of an artificial result.

Borrowing tools from semiconductor engineering, Jay Groves, assistant professor of chemistry at the University of California and his colleagues have developed a way to study receptor interactions without changing their chemistry. "We've turned to an inorganic nanotechnology

\section{BOX 1 INFORMATION OVERLOAD}

In the early days of molecular biology, signal transduction diagrams resembled chemical reactions: event $\mathrm{A}$ yields result $\mathrm{B}$. By the time the human genome sequencing project got underway, signal transduction diagrams looked more like subway maps, with reversible arrows, intersection points and complex branching patterns. Today, the same pathways are drawn like blueprints for computer chips, as researchers discover ever more sophisticated sequences of inputs, outputs and decision-making algorithms.

The point of science is to learn more about the natural world, of course, but in cell signaling, the flood of information can be overwhelming. Besides the sheer volume of findings emerging from proteomic-scale projects, researchers face a Babel of conflicting data standards. "Things haven't been so standardized, but I do think that will need to be done. There's going to need to be central repositories for storing this information," says Michael Snyder, professor of molecular, cellular and developmental biology at Yale University.

Unfortunately, neither government nor industry efforts at standardization have had much success so far. Several companies have tried to collect and distribute standardized signal transduction data, but a shortage of customers willing to pay for such a service has often made this approach unprofitable. A major governmentsupported effort, the Biomolecular Interaction Network Database (BIND) also proved unsustainable.

'These 'omic' studies are just going to pile up the data in a lot of different organisms," says Snyder, but he and other researchers are optimistic that the signal transduction field will eventually get its notebooks in order. Standard formats are now emerging for microarray data, for example, so database builders may soon catch up to proteomics and cell signaling as well. 
to try to physically rearrange molecules in a cell and create what we're now calling spatial mutations. They're chemically identical, but they're spatially different," says Groves.

To demonstrate the system, the researchers focused on the immunological synapse, the cluster of receptors responsible for triggering $\mathrm{T}$-cell maturation in the immune system. Attaching purified immunological synapse proteins to a membrane-like surface, Groves and his colleagues recreated the complexes artificially. The proteins could move freely in the artificial membrane, just as if they were on a cell surface. By installing tiny fences on the surface, the scientists could then restrict the movement of complexes to particular areas, interfering with natural complex movement in defined ways without chemically modifying the proteins themselves. Conventional signaling assays revealed which spatial interactions were important for immunological synapse signaling.

Groves says the basic technique is not difficult, and it could be applied to a wide range of receptor signaling systems using either purified proteins or crude cell membrane preparations. "We're going to have a lot of competitors soon, I'm afraid. Any university that has a reasonably sized engineering department almost certainly has [nanotechnology] fabrication facili- ties, which are primarily applied to semiconductors," says Groves.

Whatever techniques they use, many cell biologists believe that more cell signaling mechanisms remain to be discovered, and even the known pathways are just beginning to reveal their full complexity. By combining newer, highercapacity methods with established tools, though, researchers are rapidly increasing the pace of discovery. It may take many more years to finish, but the full story of cell signaling will be well worth telling.

Alan Dove is a freelance writer based in New York City (alanwdove@earthlink. net). 
TECHNOLOGY FEATURE

\section{SUPPLIERS GUIDE: COMPANIES OFHERING BQUIPMENT AND RHAGENIS FOR CHLL SIGNALING ASSAYS}

\section{Company}

AnaSpec

Applied Biosystems

BD Biosciences (Pharmingen)

Biaffin GmbH

Bio-Rad

Biotrend Chemikalien GmbH

BioVision AG

Cambrex

Chemicon

Cell Signaling Technology

CGI Pharmaceuticals

Cue BIOtech

DiscoveRx

EMD Biosciences

Exalpha Biologicals

GE Healthcare (formerly Amersham)

Globozymes

Imgenex

Intrexon

Invitrogen

Jerini AG

Kinasource

Kinexus Bioinformatics

MBL International

Molecular Devices

New England Biolabs

Olympus

Pepscan Systems

PerkinElmer

Pierce Biotechnology

Phosphosolutions

Promega

Qiagen

Rockland Immunochemicals

Serologicals

Sigma-Aldrich

Stratagene

Stressgen

Systat

Upstate

USBiological

\section{Web address}

http://www.anaspec.com/

http://www.appliedbiosystems.com/

http://www.bdbiosciences.com/pharmingen/

http://www.biaffin.com/

http://www.bio-rad.com/

http://www.biotrend.com/

http://www.biovision-discovery.de

http://www.cambrex.com/default.asp

http://www.chemicon.com/

http://www.cellsignal.com

http://www.cellulargenomics.com/

http://www.cuebiotech.com/

http://www.discoverx.com/

http://www.emdbiosciences.com/home.asp

http://www.exalpha.com/

http://www4.amershambiosciences.com/

http://www.globozymes.com/

http://www.imgenex.com/

http://www.intrexon.com/home.cfm

http://www.invitrogen.com/

http://www.jerini.de/

http://www.kinasource.co.uk/

http://www.kinexus.ca/

http://www.mblintl.com/mbli/index.asp

http://www.moleculardevices.com/

http://www.neb.com/

http://www.olympus-global.com/en/global/

http://www.pepscan.nl/

http://www.perkinelmer.com/

http://www.piercenet.com/

http://www.phosphosolutions.com/

http://www.promega.com/

http://www1.qiagen.com/

http://www.rockland-inc.com/

http://www.serologicals.com/

http://www.sigmaaldrich.com/

http://www.stratagene.com/

http://antibody.stressgen.com/

http://www.systat.com/products/SigmaPlot/

http://www.upstate.com/

http://www.usbio.net/ 